\title{
Deep Vein Thrombosis as a Rare Presentation in a Female With Anti-Neutrophil Cytoplasmic Antibodies-Associated Vasculitis
}

\author{
Wan Syamimee WAN GHAZALI, ${ }^{1}$ Nurashikin MOHAMMAD, ${ }^{1}$ Asmahan Mohd ISMAIL ${ }^{2}$ \\ ${ }^{1}$ Department of Internal Medicine, University Sains Malaysia, Kubang Kerian, Malaysia \\ ${ }^{2}$ Hospital Raja Perempuan Zainab 2, Medical, Kota Bharu, Malaysia
}

\begin{abstract}
This article aims to report a case of a young female patient with anti-neutrophil cytoplasmic antibodies-associated vasculitis complicated with pulmonary renal syndrome, multiple relapses, and who later developed venous thromboembolism. Pulmonary renal syndrome is a wellrecognized and lethal complication; however, incidence of venous thromboembolism has not been well-described. In this article, we described a 38-year-old Malay female patient admitted in 2008 with three-month history of peripheral neuropathy of lower limbs and right ankle ulcers. Initial inflammatory markers were high and perinuclear Anti-Neutrophil Cytoplasmic Antibodies were positive. She was diagnosed as anti-neutrophil cytoplasmic antibodies-associated vasculitis and started on intravenous methylprednisolone with methotrexate. She presented with relapse of skin vasculitis complicated with pulmonary renal syndrome after being stable for one year. She was intubated and proceeded with plasmapheresis and hemodialysis. She completed six cycles of cyclophosphamide. Renal biopsy revealed chronic changes consistent with end stage renal disease. She further relapsed in 2011 with nasal blockage, epistaxis, and nasal deviation. Chest X-ray revealed lung nodules. Prednisolone was increased, her symptoms settled, and she was discharged with azathioprine. She was readmitted at the end of the same year due to two-day history of right deep vein thrombosis and she later succumbed to methicillin-resistant Staphylococcus aureus sepsis.

Keywords: Anti-neutrophil cytoplasmic antibodies-associated vasculitis; deep vein thrombosis; pulmonary renal syndrome; venous thromboembolism.
\end{abstract}

Anti-neutrophil cytoplasmic antibody-associated vasculitis (AAV) is a rare but severe disease. Pulmonary renal syndrome (PRS) hemorrhage is a well-known but lethal complication. The incidence rate of PRS in patients with AAV is about 40 to $90 \%{ }^{1}$ Venous thromboembolism (VTE) has not been well-described as compared to PRS. Reported rate of mortality in AAVrelated VTE is about 0 to $15.4 \%$ and most cases have died of right heart failure as a complication of fulminant pulmonary embolism. ${ }^{2,3}$ Treatment outcome and long-term prognosis are related to early diagnosis, proper assessment of disease activity, and adequate induction and remission therapy.

In this article, we described a female patient affected by anti-neutrophil cytoplasmic antibodies
(ANCA)-associated vasculitis who developed PRS and multiple episodes of relapses. Despite aggressive immunosuppression, she later presented with two-day history of deep vein thrombosis (DVT). It is important to highlight the presence of DVT as an indicator of active disease. Despite early anticoagulant therapy for DVT, she succumbed to the complications of the disease and the treatment.

\section{CASE REPORT}

A 38-year-old Malay female patient first admitted in 2008 with numbness and weakness of both lower limbs for three months duration. It was associated with arthralgia, right ankle

Received: June 20, 2016 Accepted: August 08, 2016 Published online: January 18, 2017

Correspondence: Wan Syamimee Wan Ghazali, MD. Department of Internal Medicine, University Sains Malaysia, 16150 Kubang Kerian, Malaysia.

Tel: +60497663000 e-mail: mimeeghazali@yahoo.com

@2017 Turkish League Against Rheumatism. All rights reserved. 
ulcers, and nail fold infarcts. There was no history of fever, alopecia or mouth ulcers. Her initial investigation results showed normal full blood count and renal function. Her erythrocyte sedimentation rate was $124 \mathrm{~mm} /$ hour and C-reactive protein was $60 \mathrm{IU} / \mathrm{L}$. Antinuclear antibody and complements were normal; however, 24-hour urine protein was $0.85 \mathrm{~g} / 24$ hour (urine volume $1500 \mathrm{~mL}$ ). Anti-neutrophil cytoplasmic antibodies were positive at $>1: 20$ with a peri-nuclear pattern. Anti-glomerular basement membrane, hepatitis screening, and cryoglobulinemia were negative. Nerve conduction study revealed peripheral neuropathy (axonal type) and skin biopsy consistent with leukocytoclastic vasculitis. She was diagnosed as ANCA-AAV and started on methylprednisolone $500 \mathrm{mg}$ daily for three days. After one month of treatment, her lower limbs' weakness improved and right ankle ulcer healed. She was discharged with $1 \mathrm{mg} / \mathrm{kg}$ body weight prednisolone and methotrexate tapering dose till $20 \mathrm{mg} /$ week. She was again presented in October 2010 with multiple palpable purpura over her lower limbs involving both feet extending to calves. There were no ulcers or digital infarcts noted. Clinically, it was tender on local

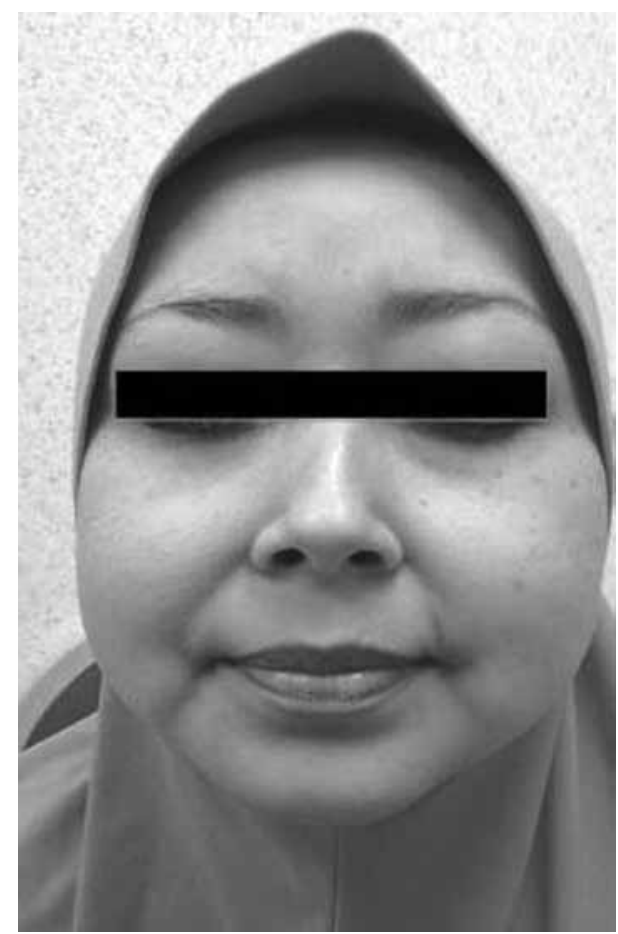

Figure 1. Clinically deviated nasal septum. palpation. Full blood count remained normal but creatinine was markedly elevated to $344 \mu \mathrm{mol} / \mathrm{L}$ from a normal baseline. During this admission, she developed PRS requiring intubation. She received five days of plasmapheresis and six days of hemodialysis with packed red blood cells. Intravenous cyclophosphamide $750 \mathrm{mg}$ and intravenous methylprednisolone $1 \mathrm{~g}$ daily for three days were also initiated. She improved symptomatically following hemodialysis and plasmapheresis. She further received intravenous pulse cyclophosphamide $750 \mathrm{mg}$ every three weeks for six cycles, with tapering dose of oral prednisolone and antihypertensive drugs. Subsequently, her renal biopsy revealed chronic change with end-stage renal disease. She was planned for renal replacement therapy but defaulted follow-up. A written informed consent was obtained from the patient.

She relapsed again in 2011, whereby at that time, she presented with two weeks history of nasal blockage, epistaxis, and fever. Clinically, her nasal septum was deviated (Figure 1). The nasal cavity was inflamed, fragile, and bled to touch. Her chest X-ray revealed well-demarcated lung lesion (Figure 2). She refused biopsy of the lung nodules. Fortunately, the lung lesion disappeared with oral prednisolone $1 \mathrm{mg} / \mathrm{kg}$ body weight. She was readmitted again after

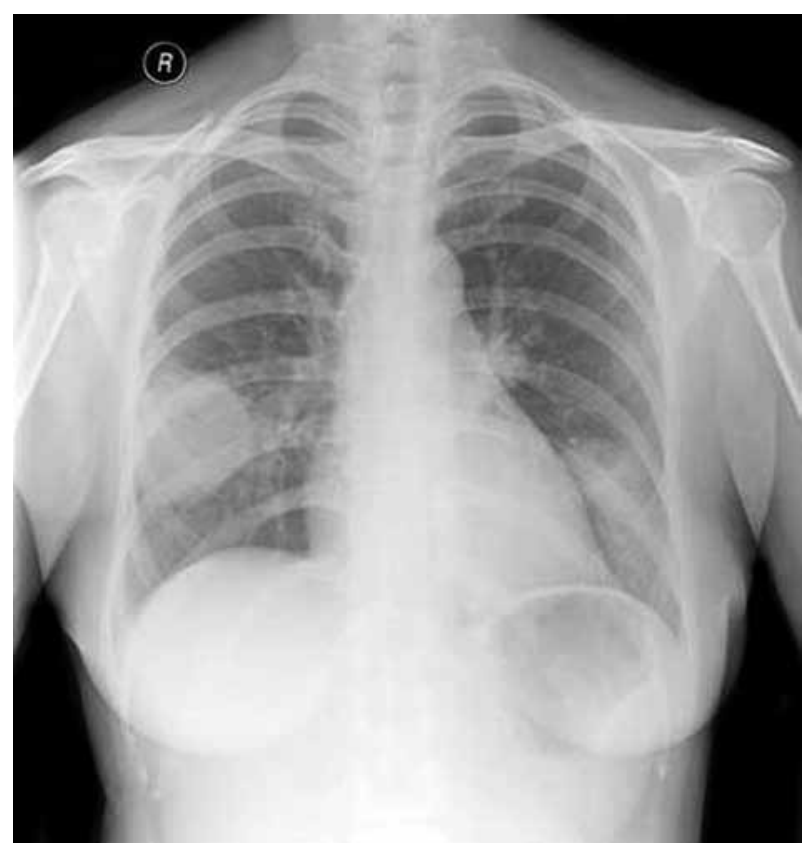

Figure 2. Bilateral well-demarcated lung nodules. 
four months due to a two-day history of swollen right lower limb. There were no histories of fever, shortness of breath, hemoptysis or cough with pleuritic chest pain. She was not cyanosed. Her blood pressure was $110 / 85 \mathrm{mmHg}$ with heart rate of $80 /$ minute and regular rhythm. Her respiratory rate was 20 per minute. Clinically, her right calf was swollen with warmth and erythema. Chest, abdominal, and neurological examinations revealed no abnormalities. There were no effusion, patch infiltrates, Westermark sign or Hampton hump. A Doppler ultrasound test of her lower limbs revealed a thrombus at right common femoral vein. Other classic risk factors for DVT were absent. Thrombophilia screening including protein $\mathrm{C}$, protein $\mathrm{S}$, lupus anticoagulant, anticardiolipin antibody, anti- $\beta 2$ glycoprotein-I antibody, and anti-thrombin III were all normal. Subcutaneous low molecular weight heparin was initiated for the DVT. However, she had complication with methicillin-resistant Staphylococcus aureus septicemia secondary to catheter related infection during her stay in the hospital. Despite good antibiotic coverage with intravenous vancomycin, she had persistent methicillin-resistant Staphylococcus aureus septicemia and succumbed after one month of hospitalization.

\section{DISCUSSION}

Among the most demanding challenges in clinical medicine is the diagnosis and management of a systemic vasculitis. The disorders themselves are rare, with an incidence of 20 to 100 cases/ million and a prevalence of 150 to $450 /$ million. $^{1-3}$ The mortality rate of AAV-associated with PRS has been reported to range from 75 to $90 \%$ in proteinase 3 (PR3)-ANCA positive and 45\% in myeloperoxidase-ANCA positive. Compared to PRS, VTE has not been well-described. Our patient demonstrated features of granulomatosis with polyangiitis with serology of microscopic polyangiitis. She was initially successfully treated with timely aggressive therapy with plasmapheresis and cyclophosphamide, but later developed VTE and succumbed to septicemia.

The incidence of VTE increases during active AAV. A prospective study by Meckel et al. ${ }^{4}$ revealed the incidence of VTE in patients with granulomatosis with polyangiitis as $7.0 / 100$ person-years compared to an incidence of $0.3 / 100$ person-years in the general. The pathogenesis of AAV-associated with VTE remains unclear. Classic risk factors for thrombo-embolic event such as immobilization, trauma, malignancy, major surgery, positive family history nor thrombophilia do not show a significant prevalence in AAV patients. Previous studies have proposed several mechanisms for VTE with AAV. Anti-neutrophil cytoplasmic antibodies stimulate neutrophilinduced cytotoxicity towards endothelial cells, leading to vessel wall inflammation and damage. Synergistic effect of infections, genetics or drugs (antibiotics, non-steroidal drugs, cyclosporine) can trigger AAV with diverse pathological roles. In addition, complementary PR3 can also be found in AAV, especially in those with PR3-ANCA. ${ }^{5}$ Complementary PR3 targets plasminogen, hence delay the conversion of plasminogen to plasmin. With increasing dissolution time of fibrin clots, complementary PR3 will result in a higher incidence of VTE. These mechanisms explain the higher risk of VTE in active AAV patients, therefore, can be an important clue for higher AAV disease activity.

The treatment with high doses of corticosteroid and cyclophosphamide may also contribute to VTE in AAV patients. Chemotherapeutic agents can induce damage to the vascular endothelium, enhanced apoptosis to endothelial cells, platelet activation and release cytokines. ${ }^{6}$

Our patient was perinuclear Anti-Neutrophil Cytoplasmic Antibodies positive. She had completed cyclophosphamide therapy and was on steroids. She developed deep vein thrombosis and later progressed to sepsis. She had both complications from her active disease as well as her treatment.

The role of prophylactic anticoagulant therapy in AAV patients remains uncertain. Currently, prophylaxis is recommended for acutely ill patients who are bedridden and have a risk for thrombosis. Randomized studies to evaluate both the protective effect of prophylaxis and the risk of bleeding should be performed as the incidence of VTE during periods of active AAV is in the same order of magnitude. ${ }^{7}$

In conclusion, introduction of aggressive immunosuppressive therapies which have been modified in recent years have an impact on 
$\mathrm{AAV}$, which used to be a deadly disease with a very high mortality rate. ${ }^{8}$ It is important to note that patients with AAV have an increased frequency of VTE. Episodes of VTE may serve as an important indicator of severe active disease. ${ }^{9}$ Anticoagulation therapy is indicated to treat and prevent recurrent episodes of this complication. The morbidity and mortality of these disorders remain alarming despite the advances made in the diagnosis and management of AAV. Treatment intervention is difficult when facing complications of active disease and the treatment itself.

\section{Declaration of conflicting interests}

The authors declared no conflicts of interest with respect to the authorship and/or publication of this article.

\section{Funding}

The authors received no financial support for the research and/or authorship of this article.

\section{REFERENCES}

1. West $\mathrm{S}$, Arulkumaran N, Ind PW, Pusey CD. Diffuse alveolar haemorrhage in ANCA-associated vasculitis. Intern Med 2013;52:5-13.

2. Stassen PM, Derks RP, Kallenberg CG, Stegeman CA. Venous thromboembolism in ANCA- associated vasculitis--incidence and risk factors. Rheumatology (Oxford) 2008;47:530-4.

3. von Scheven E, Lu TT, Emery HM, Elder ME, Wara DW. Thrombosis and pediatric Wegener's granulomatosis: acquired and genetic risk factors for hypercoagulability. Arthritis Rheum 2003;49:862-5.

4. Alpigiani MG, Calcagno A, Salvati P, Rossi GA, Barbano G, Ghiggeri G, et al. Late onset of pANCA renal and pulmonary vasculitis in a girl affected by undifferentiated connective tissue disease. Lupus 2010;19:655-7.

5. Bautz DJ, Preston GA et al. Antibodies with dual reactivity to plasminogen and complementary PR3 in PR3- ANCA vasculitis. J Am Soc Nephrol. 2008; 19(12):2421-9

6. Haddad TC, Greeno EW. Chemotherapy-induced thrombosis. Thromb Res 2006;118:555-68.

7. Geerts WH, Pineo GF, Heit JA, Bergquist D, Lassen MR, Colwell CW, et al. Prevention of venous thromboembolism: the Seventh ACCP Conference on Antithrombotic and Thrombolytic Therapy. Chest 2004;126(3 Suppl):338S-400S.

8. Ntatsaki E, Mooney J, A. Watts R. ANCA vasculitis: time for a change in treatment paradigm ? Not yet. Rheumatology 2011 ; 50: 1019- 1024

9. Merkel PA, Lo GH, Holbrook JT, Tibbs AK, Allen NB, Davis JC Jr, et al. Brief communication: high incidence of venous thrombotic events among patients with Wegener granulomatosis: the Wegener's Clinical Occurrence of Thrombosis (WeCLOT) Study. Ann Intern Med 2005;142:620-6. 